PROCEEDINGS OF THE

AMERICAN MATHEMATICAL SOCIETY

Volume 127, Number 12, Pages 3513-3516

S 0002-9939(99)05081-9

Article electronically published on May 13, 1999

\title{
A MORPHISM OF INTERSECTION HOMOLOGY INDUCED BY AN ALGEBRAIC MAP
}

\author{
ANDRZEJ WEBER
}

(Communicated by Leslie Saper)

\begin{abstract}
Let $f: X \rightarrow Y$ be a map of algebraic varieties. Barthel, Brasselet, Fieseler, Gabber and Kaup have shown that there exists a homomorphism of intersection homology groups $f^{*}: I H^{*}(Y) \rightarrow I H^{*}(X)$ compatible with the induced homomorphism on cohomology. The crucial point in the argument is reduction to the finite characteristic. We give an alternative and short proof of the existence of a homomorphism $f^{*}$. Our construction is an easy application of the Decomposition Theorem.
\end{abstract}

Let $X$ be an algebraic variety, $I H^{*}(X)=H^{*}\left(X ; I C_{X}\right)$ its rational intersection homology group with respect to the middle perversity and $I C_{X}$ the intersection homology sheaf which is an object of derived category of sheaves over $X$ [GM1]. We have the homomorphism $\omega_{X}: H^{*}(X ; \mathbb{Q}) \longrightarrow I H^{*}(X)$ induced by the canonical morphism of the sheaves $\omega_{X}: \mathbb{Q}_{X} \longrightarrow I C_{X}$.

Let $f: X \longrightarrow Y$ be a map of algebraic varieties. It induces a homomorphism of the cohomology groups. The natural question arises: Does there exist an induced homomorphism for intersection homology compatible with $f^{*}$ ?

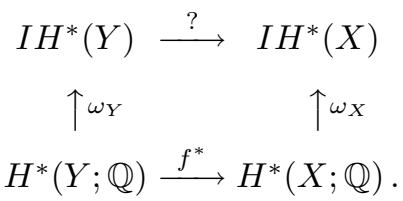

The answer is positive. For topological reasons the map in question exists for normally nonsingular maps [GM1, §5.4.3] and for placid maps [GM3, §4]. The authors of $[\mathrm{BBFGK}]$ proved the following:

Theorem 1. Let $f: X \longrightarrow Y$ be an algebraic map of algebraic varieties. Then there exists a morphism $\lambda_{f}: I C_{Y} \longrightarrow R f_{*} I C_{X}$ such that the following diagram with the canonical morphisms commutes:

$$
\begin{gathered}
I C_{Y} \stackrel{\lambda_{f}}{\longrightarrow} R f_{*} I C_{X} \\
\uparrow \omega_{Y} \quad \uparrow R f_{*}\left(\omega_{X}\right) \\
\mathbb{Q}_{Y} \stackrel{\alpha_{f}}{\longrightarrow} R f_{*} \mathbb{Q}_{X} .
\end{gathered}
$$

Received by the editors February 24, 1998.

1991 Mathematics Subject Classification. Primary 14F32, 32S60; Secondary 14B05, 14C25.

Key words and phrases. Intersection homology, algebraic varieties, morphism.

The author was partially supported by KBN 2 P03A 01113 grant.

(C)1999 American Mathematical Society 
In fact, $[\mathrm{BBFGK}]$ proves the existence of a morphism $\mu_{f}: f^{*} I C_{Y} \rightarrow I C_{X}$, which is adjoint to $\lambda_{f}$.

The sheaf language can be translated to the following: an induced homomorphism of intersection homology exists in a functorial way with respect to the open subsets of $Y$. This means that there exists a compatible family of induced homomorphisms

$$
f_{\lambda, U}^{*}: I H^{*}\left(f^{-1} U\right) \longrightarrow I H^{*}(U)
$$

which is also compatible with the family

$$
f_{\mid U}^{*}: H^{*}\left(f^{-1} U ; \mathbb{Q}\right) \longrightarrow H^{*}(U ; \mathbb{Q}) .
$$

As shown in [BBFGK] the morphism $\lambda_{f}$ (and $\mu_{f}$ ) is not unique. It is not possible to choose the morphisms $\lambda_{f}$ (or $\mu_{f}$ ) in a functorial way with respect to all algebraic maps (p.160). The simplest counterexample is the inclusion $\{(0,0)\} \hookrightarrow\left\{\left(x_{1}, x_{2}\right)\right.$ : $\left.x_{1} x_{2}=0\right\}$, which can be factored through the inclusions $\{(0,0)\} \hookrightarrow\left\{\left(x_{1}, x_{2}\right)\right.$ : $\left.x_{i}=0\right\}$ for $i=1$ or 2 .

We will give a short proof of the main theorem from [BBFGK]. We will derive it from the Decomposition Theorem. The reference to the Decomposition Theorem is [BBD, 6.2.8] (see also [GM2]) and in a slightly different context [Sa].

We will use only the following corollary from the Decomposition Theorem:

Corollary from the Decomposition Theorem. Let $\pi: X \longrightarrow Y$ be a proper surjective map of algebraic varieties. Then $I C_{Y}$ is a direct summand in $R \pi_{*} I C_{X}$.

The idea of the proof of our theorem is simple; the essence is the argument similar to [BBFGK, Remarque pp.172-174]. We take a resolution $\pi_{Y}: \widetilde{Y} \longrightarrow Y$ and enlarge the space $X$ to obtain a map $\tilde{f}: \widetilde{X} \longrightarrow \widetilde{Y}$. There exists the induced morphism of intersection homology $\lambda_{\tilde{f}}$ for $\tilde{f}$. By the Decomposition Theorem the intersection homology of $X$ (and $Y$ ) is a direct summand of intersection homology of $\widetilde{X}$ (resp. $\widetilde{Y}$ ). We compose $\lambda_{\tilde{f}}$ with the projection and inclusion in the direct sums to obtain the desired morphism $\lambda_{f}$.

Remark. If we insisted, then $\widetilde{X}$ might be even smooth of the same dimension as $X$ with the map $\pi_{X}: \widetilde{X} \longrightarrow X$ generically finite; compare [BBFGK, p.173].

Proof of Theorem 1. We may assume that $X$ and $Y$ are irreducible. Let $\pi_{Y}: \widetilde{Y} \longrightarrow$ $Y$ be a resolution of Y. Denote by $\widetilde{X}$ the fiber product (pull-back) $X \times_{Y} \widetilde{Y}$. Note that it is a variety, which may be singular and not equidimensional. We have a commutative diagram of algebraic maps $\left(\pi_{X}\right.$ and $\pi_{Y}$ proper):

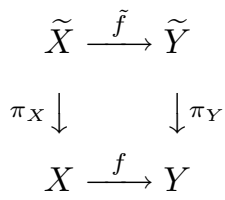

and the associated diagram of sheaves over $Y$ :

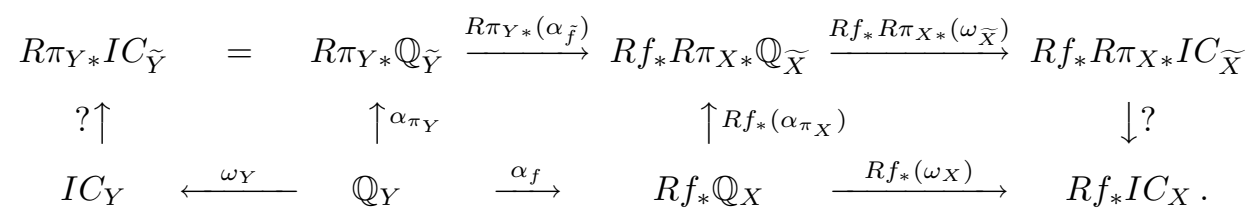


To prove the existence of a morphism $\lambda_{f}: I C_{Y} \longrightarrow R f_{*} I C_{X}$, we will show that the arrows with question marks exist in a way that the diagram remains commutative. The existence of such morphisms follows from the Decomposition Theorem for $\pi_{Y}$ and $\pi_{X}$ (see the corollary). The sheaf $I C_{Y}$ is a direct summand in $R \pi_{Y *} I C_{\tilde{Y}}$ :

$$
i: I C_{Y} \hookrightarrow R \pi_{Y *} I C_{\widetilde{Y}} .
$$

We also have a projection:

$$
p: R \pi_{X *} I C_{\widetilde{X}} \longrightarrow I C_{X}
$$

which induces

$$
R f_{*}(p): R f_{*} R \pi_{X *} I C_{\widetilde{X}} \longrightarrow R f_{*} I C_{X} .
$$

It remains to prove the commutativity of the diagram. We compare the morphisms over $Y$ :

$$
\mathbb{Q}_{Y} \stackrel{\omega_{Y}}{\longrightarrow} I C_{Y} \stackrel{i}{\longrightarrow} R \pi_{Y *} I C_{\widetilde{Y}}=R \pi_{Y *} \mathbb{Q}_{\tilde{Y}}
$$

and the natural one

$$
\mathbb{Q}_{Y} \stackrel{\alpha_{\pi_{Y}}}{\longrightarrow} R \pi_{Y *} \mathbb{Q}_{\tilde{Y}}
$$

Respectively over $X$ we compare the morphisms:

$$
\mathbb{Q}_{X} \stackrel{\alpha_{\pi_{X}}}{\longrightarrow} R \pi_{X *} \mathbb{Q}_{\widetilde{X}} \stackrel{R \pi_{X *}\left(\omega_{\widetilde{X}}\right)}{\longrightarrow} R \pi_{X *} I C_{\widetilde{X}} \stackrel{p}{\longrightarrow} I C_{X}
$$

and the canonical one

$$
\mathbb{Q}_{X} \stackrel{\omega_{X}}{\longrightarrow} I C_{X} .
$$

Let $U$ (resp. $V$ ) be the regular part of $Y$ (resp. $X$ ). After multiplication by a constant if necessary, these morphisms are equal on $U$ (resp. on $V$ ). We will show that an equality of morphisms over an open set implies the equality over the whole space. We have the restriction morphism

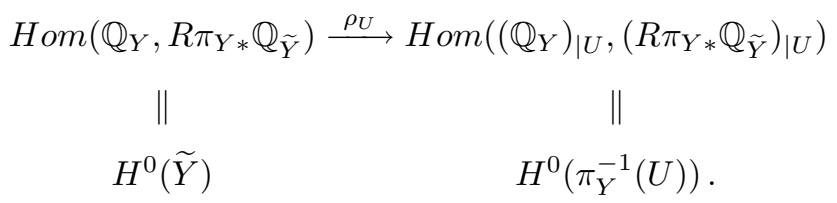

The kernel of $\rho_{U}$ is $H^{0}\left(\widetilde{Y}, \pi_{Y}^{-1}(U)\right)$, which is trivial. We have the same for the morphisms over $X$ :

$$
\begin{array}{cc}
\operatorname{Hom}\left(\mathbb{Q}_{X}, I C_{X}\right) \stackrel{\rho_{V}}{\longrightarrow} \operatorname{Hom}\left(\left(\mathbb{Q}_{X}\right)_{\mid V},\left(I C_{X}\right)_{\mid V}\right) \\
\| & \| \\
I H^{0}(X) & I H^{0}(V) .
\end{array}
$$

The kernel is $I H^{0}(X, V)=0$.

Remark. The restriction morphisms $\rho_{U}$ and $\rho_{V}$ are in fact isomorphisms. The cokernel of $\rho_{U}$ is contained in $H^{1}\left(\widetilde{Y}, \pi_{Y}^{-1}(U)\right)=H_{2(\operatorname{dim} \tilde{Y})-1}^{c l}\left(\widetilde{Y} \backslash \pi_{Y}^{-1}(U)\right)$ which is trivial for dimensional reasons. The second follows from [Bo, V.9.2 p.144] as noticed in [BBFGK, p.178]. 


\section{ACKNOWLEDGEMENT}

I would like to acknowledge the hospitality of the Institut de Mathèmatiques de Luminy and to thank Prof. J. P. Brasselet personally for the encouragement to find a simpler proof of the main theorem from [BBFGK]. I would also like to thank the authors of $[\mathrm{BBFGK}]$ for correcting this note.

\section{REFERENCES}

[Bo] A. Borel (ed.), Intersection cohomology, Progress in Mathematics Vol. 50 (A. Borel, ed.), Birkhäuser, 1984. MR 88d:32024

[BBD] A. Beilinson, J. Bernstein, P. Deligne, Faisceaux Pervers, Astérisque 100 (1983). MR 86g:32015

[BBFGK] G. Barthel, J.-P. Brasselet, K.-H. Fieseler, O. Gabber, L. Kaup, Relèvement de cycles algébriques et homomorphismes associés en homologie d'intersection, Ann. Math 141 (1995), 147-179. MR 96a:14027

[GM1] M. Goresky, R. MacPherson, Intersection homology II, Invent. Math. 72 (1983), 77-130. MR 84i: 57012

[GM2] M. Goresky, R. MacPherson, On the topology of complex algebraic maps, Geometry La Rabida, Lecture Notes in Mathematics, vol. 961, Springer Verlag, N. Y., 1982, pp. 119-129. MR 85f:32019

[GM3] M. Goresky, R. MacPherson, Lefschetz fixed point theorem for intersection homology, Comm. Math. Helv. 60 (1985), 366-391. MR 87f:32030

[Sa] M. Saito, Decomposition theorem for proper Kähler morphisms, Tôhoku Math. J. 42 (1990), 127-148. MR 91j:32042

Institute of Mathematics, Warsaw University, ul. Banacha 2, 02-097 Warszawa, POLAND

E-mail address: aweber@mimuw.edu.pl 